\title{
Replication of psychometric properties and predictive validity of the Important People Drug and Alcohol Interview
}

\author{
Mandy D. Owens ${ }^{1}$ and William H. Zywiak ${ }^{2}$ \\ ${ }^{1}$ Center on Alcoholism, Substance Abuse, and Addictions, University of New Mexico, Albuquerque, New Mexico, United States \\ ${ }^{2}$ Decision Sciences Institute, Pacific Institute for Research and Evaluation, Pawtucket, Rhode Island, United States and Department of Psychiatry \\ and Human Behavior, Center for Alcohol and Addiction Studies, Brown University, Providence, Rhode Island, United States
}

\begin{abstract}
Aims: Social support is a predictor of alcohol and drug use. The Important People Drug and Alcohol (IPDA) interview and its predecessor, the Important People and Activities (IPA) measure, have been used to demonstrate this predictive relationship. The purpose of this study was to replicate the findings from Zywiak et al. (2009) in a sample of probationers with substance use disorders.

Design: Analyses mirrored those done previously to replicate the associations between social networks and substance use. The IPDA was used to assess social networks before and after incarceration. Form-90 (Tonigan, Miller, \& Brown, 1997) was used to measure substance use.

Participants: Individuals were recruited from a local probation office. Information was collected from a sample of 50 male probationers with substance use disorders recently released from jail.

Conclusions: Results showed that many of the previous findings from Zywiak et al. (2009) were similar to those found in the current study. This adds to the evidence that the IPDA is a promising measure of social networks and examining how those networks relate to substance use outcomes. The use of the IPDA may be beneficial for both research and clinical purposes, while evaluating individuals with alcohol and other drug use disorders.
\end{abstract}

The Important People and Activities (IPA) measure was administered longitudinally in the two largest randomized clinical trials of alcohol treatment conducted to-date in the United States (Project COMBINE, Anton et al., 2006; Project MATCH, Project MATCH Research Group, 1997). For each participant, the IPA measure collects a list of people important to the participant and a variety of characteristics for each person identified, including: frequency of contact; alcohol use status; alcohol use frequency; support for alcohol; and general support (a modified version of this measure was used in the present study and is described in detail in the Measures section). The most widespread clinical application of this measure was evident in the Combined Pharmacotherapies and Behavioral Interventions (COMBINE) study (Project COMBINE, Anton et al., 2006). Five of the nine cells in the research design implemented the Combined Behavioral Intervention (CBI; Miller, 2004); the IPA measure was incorporated into this intervention in three ways. First, it provided preliminary data to help the therapist and client identify a supportive significant other who was then invited to attend sessions with the client. Second, the IPA measure was used more integrally in the social and recreational counseling component of the CBI (e.g., “... it sounds like many of your regular contacts were drinking companions. One of the important challenges is to develop new interests, friends, and rewarding ways to spend your time that don't involve alcohol” (Miller, 2004, p. 198)). Third, the IPA measure was used again in the social support for sobriety component of CBI. Here, depending on the client's network, three types of social support problems may be tagged and addressed: clients with few or no general support measures, clients with low support for abstinences, and clients with high network support for drinking (Miller, 2004, pp. 203-204).

To date, the IPA family of measures have been used more widely as research tools rather than clinical tools. When used empirically, the raw data is used to compute a number of indices. For example, in Project MATCH $(n=1,726)$ the social network support for drinking variable (a composite of 11 different IPA indices) exhibited a matching effect. More specifically, clients with high support for drinking in the outpatient arm had better shortterm (3 to 4 weeks) and long-term outcomes (months 37 through 39) if randomized to Twelve Step Facilitation

Correspondence: Mandy D. Owens, Department of Psychology, University of New Mexico, MSC03 2220, Albuquerque, NM 87106. E-mail: mandyo@unm.edu Financial support: This research was supported by internal grants from the Office of Graduate Studies, Graduate and Professional Student Association, and the Department of Psychology at the University of New Mexico; and NIAAA grant T32-AA018108.

Keywords: social support; alcohol and drug use; probationers; criminal justice system; measurement 
rather than Motivational Enhancement Therapy (Project MATCH Research Group, 1998a; 1998b). These 11 indices were: number of network members; number of members with daily contact; average importance of network members; network members' drinking status, frequency, and maximum drinks per drinking episode; proportion of heavy drinkers in the network; and the most important network members' reactions to the clients' drinking - which made up the last three indices: most support, least support, and average support for drinking (Zywiak, Longabaugh, \& Wirtz, 2002). Additionally, during the 12 months following treatment, the social network support for drinking variable demonstrated prognostic effects on percentage days abstinent in the outpatient arm and on drinks per drinking day in the aftercare arm (Project MATCH Research Group, 1997). Network support for drinking variables from a later generation IPA measure also demonstrated prognostic effects on drinking outcomes (Longabaugh, Wirtz, Zywiak, \& O'Malley, 2010); this is exemplified in Project COMBINE (Anton et al., 2006), which enrolled 1,373 alcohol dependent clients, and examined the unique and combined effects of psychosocial and pharmacological interventions.

In Project MATCH, the 11 indices used to compute the single matching variable showed poor convergent validity (Zywiak et al., 2002). Indices from the Important People Drug and Alcohol (IPDA) interview, adapted by Dr. Neighbors for use in a cocaine dependence treatment outcome study $(n=141)$, showed much better internal consistency (Zywiak et al., 2009). More specifically, substance involvement (e.g., status, frequency of use), general/treatment support, and support for substance use exhibited Cronbach's alpha of .92, .84, and .85 respectively. This measure also demonstrated predictive validity and matching results. Given the encouraging findings from Zywiak and colleagues (2009), it may be helpful to replicate their results using a new study sample and the IPDA measure. The aims of the current study were to: (a) replicate the analyses examined by Zywiak et al. (2009) in a new sample of individuals with alcohol and other substance use disorders, (b) compare the results from these analyses with the results found by Zywiak et al. (2009), and (c) examine the predictive validity of the three indices and three component scores using the analyses performed by Zywiak et al. (2009).

\section{Methods}

\section{Participants}

Participants came from a study conducted by Owens and McCrady (2014) that examined the association between social networks and substance use for probationers recently released from jail. Fifty adult male probationers were recruited via flyers and posters at a large Probation and Parole Division office in New Mexico from October 2011 to February 2013. Interested individuals were screened over the phone and were included in the study if they met these criteria: recently (60 to 210 days prior to screening) were incarcerated in jail for an alcohol or drug-related offense (e.g., driving under the influence, possession of illicit drugs, probation violation related to a positive urine drug screen); were currently were on probation; had moderate or high substance use involvement prior to incarceration as measured by the National Institute on Drug Abuse-Modified Alcohol, Smoking, and Substance Involvement Screening Test (Hides et al., 2009); and used alcohol or drugs during the 30 days prior to incarceration. Exclusion criteria included: if the interested individual's prior incarceration was in prison (not jail) to exclude those with longer-term incarceration ( $>1$ year), if the individual was not fluent in English because translated measures and bilingual research staff were not available, if the prior incarceration was less than six days to exclude those who might still have been in withdrawal from alcohol or drugs after release, and if the individual exhibited current psychotic symptoms as measured by the Structured Clinical Interview for the Diagnostic Statistic Manual of Mental Disorders Fourth Edition (First, Spitzer, Gibbons, \& Williams, 2002). Table 1 provides a description of participants, including demographic, incarceration, and substance use information.

\section{Procedures}

All procedures were approved by the institutional review board at the University of New Mexico and were consistent with the principles outlined in the Declaration of Helsinki of 1975. After screening, eligible individuals provided informed consent and participated in a single, in-person assessment interview where they were compensated with a \$25 store gift card. At this interview, participants completed measures that assessed social network and substance use information from the 30 days prior to incarceration (T1), 30 days after release from jail (T2), and from the 31 days after release from jail until the day prior to the assessment interview (T3; duration of T3: $M=96.2$, $S D=33.6$, range $=41-176$ days). Primary drugs of use, as determined by frequency of use prior to incarceration, were: $47 \%$ alcohol, $20 \%$ marijuana, $18 \%$ opiates, $10 \%$ cocaine, and $4 \%$ methamphetamine. Owens and McCrady (2014) provide additional detailed information regarding participants and study procedures.

\section{Measures}

To measure social networks, the IPDA measure was administered with three modifications. First, participants were permitted to list children of all ages on their list of up to 12 social network members. Second, participants were asked to rate network members' reaction to their substance use, in general. This modification differed from the IPDA in that it asked for a single rating of network members' reaction to the participant's alcohol and drug use, rather than a separate rating for alcohol and a separate rating for drug use. Last, three additional questions were added which assessed network members' substance of choice (i.e., alcohol, marijuana, cocaine, opioids, methamphetamine, poly-user, or other), and how often the participant used alcohol and how often the participant used drugs with each network member on a 7-point scale $(0=$ not at all during this time period, $7=$ daily). The three indices used were 
total network size, daily network size, and average importance of network members.

Three components were also computed: substance involvement, general/treatment support, and support for substance use. The substance involvement component was computed by weighting the substance involvement items by the contact frequency for each network member. The general/treatment support component was similarly computed by weighting general support and treatment support across the network members, and combining these terms. Support for substance use was computed by weighting the support for substance use by the contact frequency of network members. Participants' social networks were assessed chronologically from T1 to T2 to T3; this was the order that data were collected for all participants. It was assumed that T3 social networks would be the easiest for participants to recall based on recency effects. To avoid the perseveration of participants' current social network into earlier social networks, T1 and T2 social networks were assessed first.

Substance use was measured using an adapted version of Form-90 (Tonigan et al., 1997), which measured the quantity and frequency of alcohol and drug use (total days of non-alcohol substance use and the number of days of use by specific type of substance) for each time period. The percentage of days abstinent (PDA) was calculated using the number of days abstinent from both alcohol and drugs divided by the total number of days in each time period. The percentage of days of heavy drinking (PDH; i.e., 5 or more standard drinks in one day) were computed based on the number of heavy drinking days divided by the total number of days in each time period (see Table 1).

Table 1

Descriptive information for study participants

\begin{tabular}{|c|c|c|}
\hline Demographic Variable & $n(\%)$ & Mean (SD) \\
\hline Male & $50(100)$ & \\
\hline \multicolumn{3}{|l|}{ Ethnicity } \\
\hline American Indian or Alaskan Native & $1(2.0)$ & \\
\hline Black or African-American & $10(20.0)$ & \\
\hline Hispanic & $28(56.0)$ & \\
\hline White, not of Hispanic origin & $8(16.0)$ & \\
\hline Multi-racial & $1(2.0)$ & \\
\hline Chose not to answer & $2(4.0)$ & \\
\hline Age (years) & & $40.8(12.4)$ \\
\hline \multicolumn{3}{|l|}{ Education } \\
\hline No degree & $21(42.0)$ & \\
\hline High school diploma & $10(20.0)$ & \\
\hline Graduate equivalent degree & $8(16.0)$ & \\
\hline Trade school certificate & $4(8.0)$ & \\
\hline Associate degree & $3(6.0)$ & \\
\hline Bachelor's degree & $1(2.0)$ & \\
\hline Master's degree & $1(2.0)$ & \\
\hline Chose not to answer & $1(2.0)$ & \\
\hline \multicolumn{3}{|l|}{ Incarceration } \\
\hline Days incarcerated & & $99.3(99.1)$ \\
\hline Days since release & & $128.7(42.5)$ \\
\hline \multicolumn{3}{|l|}{ Substance Use } \\
\hline T1 $\mathrm{PDA}^{\mathrm{a}}$ & & $37.8(38.0)$ \\
\hline $\mathrm{T} 2 \mathrm{PDA}^{\mathrm{b}}$ & & $73.3(36.6)$ \\
\hline T3 PDA $^{\mathrm{c}}$ & & $79.1(33.2)$ \\
\hline $\mathrm{T} 1 \mathrm{PDH}^{\mathrm{b}}$ & & $26.7(35.2)$ \\
\hline $\mathrm{T} 2 \mathrm{PDH}^{\mathrm{b}}$ & & $8.8(21.2)$ \\
\hline T3 PDH & & $5.2(12.6)$ \\
\hline \multicolumn{3}{|l|}{ Indices } \\
\hline Network size (square root) & & $2.5(0.6)$ \\
\hline Daily network size & & $3.0(2.1)$ \\
\hline Importance of most important people & & $4.6(1.1)$ \\
\hline
\end{tabular}

Notes. $\mathrm{T} 1=$ the 30 days prior to incarceration; $\mathrm{T} 2=$ the 30 days after incarceration; $\mathrm{T} 3=31$ days after incarceration to the day before the assessment interview. Importance of social network members was rated on a Likert-type scale, where $4=$ "Important" and $5=$ "Very Important." ${ }^{\mathrm{a}} n=48{ }^{\mathrm{b}} n=49{ }^{\mathrm{c}} n=47$ 
Similar to the procedure for the IPDA in this study, substance use was measured retrospectively for T1, T2, and T3. Participants also provided urine samples, which were tested for recent drug use. Because urine samples were tested on site, analyses that indicated an "invalid" result $(n=3)$ were retested again until urine results were valid. Only one participant refused to do the urine analysis. Results were consistent with previous studies (e.g., Napper, Fisher, Johnson, \& Wood, 2010; Project MATCH Research Group, 1997; Weiss et al., 1998), in that there were no discrepancies between urine analysis results and selfreported substance use.

\section{Analysis Approach}

All analyses were conducted using SPSS version 20 (IBM Corporation, 2011). Three stand-alone indices and three composite scores from the IPDA for T1 were computed based on the scoring methods reported by Zywiak et al. (2009) to test if their results replicated in the current sample of male probationers. Index 1 (network size) was a square root transformation of participants' social network size (up to 12 members); transformation helps produce a more normally distributed variable. Index 2 (daily network size) was a total of network members with whom participants had daily contact. Index 3 (importance) was an average rating of importance of the four most important people in the participants' social network $(1=$ not at all important, $6=$ extremely important). The three indices were used since they reflect social investment and because they have been important predictors of alcohol use (Zywiak et al., 2002; Zywiak et al., 2009) and drug outcomes (Zywiak et al., 2009).

Component 1, substance use involvement ( $\alpha=.96$; cf to .92 in Zywiak et al., 2009), included measures of network members' alcohol use status and frequency of use, together with members' drug use status and frequency of use. Component 2, general/treatment support ( $\alpha=.83$; cf to .84 in Zywiak et al., 2009), incorporated the maximum, average, and minimum ratings of general support; as well as the maximum, average, and minimum ratings of support for substance abuse treatment. Component 3, support for substance use, ( $\alpha=.75$; cf to .85 in Zywiak et al., 2009), consisted of a maximum and average rating of support substance use. Means and standard deviations for the Component scores are not reported because these scores are based on $z$ scores that were calculated for each individual and then averaged across the sample $(M=0$ and $S D=1$ for all Component scores).

Analyses presented were conducted to attempt to replicate analyses reported by Zywiak et al. (2009). First, after the indices and component values were computed for the IPDA, intercorrelations were calculated among these six scores. Next, the three index and three component scores from $\mathrm{T} 1$ were correlated with the number of days of drug use, the number of days of alcohol use, and the number of days of complete abstinence during $\mathrm{T} 1$. Then, the three index and the three component scores from $\mathrm{T} 1$ were correlated with the number of days of drug use, the number of days of alcohol use, and the number of days of complete abstinence during $\mathrm{T} 2$ and $\mathrm{T} 3$; this tested the predictive validity of these indices and component scores. In the original Zywiak et al. (2009) paper, the substance use involvement component did not predict subsequent alcohol and drug use. For the current study, replication of the "post-hoc analyses" by Zywiak and others (2009) consisted of a repeated measures ANOVA, testing the main effects of relapse groups (i.e., individuals who used any alcohol or drugs in $\mathrm{T} 2$ or $\mathrm{T} 3$ versus those who abstained) and the interaction of relapse group by time for Component 1 (i.e., substance use involvement) scores. The main effect results tested if Component 1 scores were consistent across time for individuals who relapsed versus those who remained abstinent after release from jail (where T2 and T3 were examined separately). The interaction of relapse group by time measured if changes in substance use involvement across $\mathrm{T} 1$ to $\mathrm{T} 2$ and $\mathrm{T} 1$ to $\mathrm{T} 3$ differed by individuals who used any alcohol or drugs during $\mathrm{T} 2$ and $\mathrm{T} 3$, respectively.

\section{Results}

\section{Preliminary Analyses}

The correlation matrix between the six IPDA variables (three indices and three component scores) in the present study is shown in Table 2 and was strikingly similar to the correlation matrix reported by Zywiak et al. (2009). Indeed, the correlation of the respective correlation values across the two studies was large, $r(13)=.83, p<.001$.

\section{Concurrent Validity Analyses}

Concurrent correlations among the six IPDA variables from T1 and substance use are shown in Table 3 . In the concurrent validity analyses, as in the prior paper, general treatment support was inversely related to drug days, and substance use involvement was directly related to drinking days. The finding that network size was inversely related to drug days was not replicated. New findings emerged, with the size of the daily network being directly related to drug days. Importance of the four most important people in the network was inversely related to drinking days, and general/treatment support was inversely related to drinking days.

\section{Predictive Validity Analyses}

Table 4 presents the correlations among the T1 IPDA variables and substance use during $\mathrm{T} 2$ and $\mathrm{T} 3$. There were differences in the prospective correlations between the current sample and those found previously. In general, a greater number of significant findings were noted in the present study compared to those found by Zywiak et al. (2009), despite a smaller sample size. Substance use involvement and support for use were directly related to T2 drug days. In addition, the daily network size was directly related T2 drug days. The network size was inversely related to $\mathrm{T} 3$ abstinent days 
Table 2

Index and component intercorrelations: Comparisons with Zywiak et al. (2009)

\begin{tabular}{|c|c|c|c|c|}
\hline & \multicolumn{2}{|c|}{ Index 2} & \multicolumn{2}{|c|}{ Index 3} \\
\hline & $\begin{array}{c}\text { Current } \\
\text { Study }\end{array}$ & $\begin{array}{c}\text { Zywiak } \\
\text { (2009) }\end{array}$ & $\begin{array}{c}\text { Current } \\
\text { Study }\end{array}$ & $\begin{array}{c}\text { Zywiak } \\
\text { (2009) }\end{array}$ \\
\hline Index 1 (network size) & $0.41^{* *}$ & $0.43 * * *$ & 0.24 & 0.06 \\
\hline Index 2 (daily network size) & & & $0.29 *$ & $0.22 * *$ \\
\hline Index 3 (importance) & & & & \\
\hline Component 1 (substance use involvement) & & & & \\
\hline Component 2 (general/treatment support) & & & & \\
\hline Component 3 (support for substance use) & & & & \\
\hline
\end{tabular}

\begin{tabular}{|c|c|c|c|c|c|c|}
\hline & \multicolumn{2}{|c|}{ Component 1} & \multicolumn{2}{|c|}{ Component 2} & \multicolumn{2}{|c|}{ Component 3} \\
\hline & $\begin{array}{c}\text { Current } \\
\text { Study }\end{array}$ & $\begin{array}{c}\text { Zywiak } \\
\text { (2009) }\end{array}$ & $\begin{array}{c}\text { Current } \\
\text { Study }\end{array}$ & $\begin{array}{c}\text { Zywiak } \\
\text { (2009) }\end{array}$ & $\begin{array}{c}\text { Current } \\
\text { Study }\end{array}$ & $\begin{array}{c}\text { Zywiak } \\
\text { (2009) }\end{array}$ \\
\hline Index 1 (network size) & $0.42 * *$ & $0.39 * * *$ & 0.06 & 0.05 & -0.25 & $0.16^{*}$ \\
\hline Index 2 (daily network size) & $0.50 * * *$ & $0.22 * *$ & 0.08 & $0.17 *$ & 0.09 & $0.15^{*}$ \\
\hline Index 3 (importance) & -0.16 & -0.02 & $0.73 * * *$ & $0.37 * * *$ & -0.13 & 0.01 \\
\hline Component 1 (substance use involvement) & & & $-0.44 * *$ & $-0.22 * *$ & $0.39 * *$ & $0.41^{* * *}$ \\
\hline Component 2 (general/treatment support) & & & & & $-0.29 *$ & $-0.36 * * *$ \\
\hline Component 3 (support for substance use) & & & & & & \\
\hline
\end{tabular}

Notes. ${ }^{*} p<.05 .{ }^{* *} p<.01 .{ }^{* *} p<.001$

Table 3

IPDA preincarceration (T1) index and component correlations with concurrent substance use

\begin{tabular}{lccc}
\hline & Drug Days & Drinking Days & Abstinent Days \\
\hline Index 1 (network size) & -0.023 & 0.010 & -0.052 \\
Index 2 (daily network size) & $0.309^{*}$ & -0.060 & -0.177 \\
Index 3 (importance) & -0.085 & $-0.459^{* *}$ & 0.216 \\
Component 1 (substance use involvement) & $0.333^{*}$ & $0.296^{*}$ & $-0.335^{*}$ \\
Component 2 (general/treatment support) & $-0.289^{*}$ & $-0.338^{*}$ & $0.354^{*}$ \\
Component 3 (support for substance use) & 0.204 & 0.224 & -0.217 \\
\hline
\end{tabular}

Notes. Component 3 included a combined measure of support for alcohol and drug use.

${ }^{*} p<.05 .{ }^{* *} p<.01$.

Table 4

IPDA pre-incarceration (T1) index and component correlations with post-incarceration (T2 and T3) substance use

\begin{tabular}{lllcccccc}
\hline & \multicolumn{3}{c}{ Post-Incarceration (T2) } & & \multicolumn{3}{c}{ Present (T3) } \\
\cline { 2 - 3 } \cline { 6 - 8 } & $\begin{array}{c}\text { Drug } \\
\text { Days }\end{array}$ & $\begin{array}{c}\text { Drinking } \\
\text { Days }\end{array}$ & $\begin{array}{c}\text { Abstinent } \\
\text { Days }\end{array}$ & & $\begin{array}{c}\text { Drug } \\
\text { Days }\end{array}$ & $\begin{array}{c}\text { Drinking } \\
\text { Days }\end{array}$ & $\begin{array}{c}\text { Abstinent } \\
\text { Days }\end{array}$ \\
\hline Index 1 (network size) & 0.126 & 0.180 & -0.166 & & 0.192 & 0.194 & $-0.312^{*}$ \\
Index 2 (daily network size) & $0.331^{*}$ & 0.014 & -0.268 & & 0.213 & 0.038 & -0.231 \\
Index 3 (importance) & 0.096 & 0.098 & -0.138 & & 0.149 & 0.112 & -0.141 \\
Component 1 (substance use involvement) & $0.293^{*}$ & 0.040 & -0.253 & & 0.189 & 0.184 & -0.140 \\
Component 2 (general/treatment support) & -0.167 & 0.084 & 0.099 & & -0.021 & 0.014 & -0.091 \\
Component 3 (support for substance use) & $0.345^{*}$ & 0.097 & $-0.300^{*}$ & & -0.055 & -0.012 & 0.246 \\
\hline
\end{tabular}

Notes. Component 3 included a combined measure of support for alcohol and drug use. $* p<.05$. 
For the present study, the post hoc analyses conducted by Zywiak and colleagues (2009) were repeated and examined the association between substance use involvement during $\mathrm{T} 1$ and relapsing at $\mathrm{T} 2$ and $\mathrm{T} 3$. Here, the results were similar to Zywiak et al. (2009) where a significant interaction was found between changes in substance use involvement and relapse status at T2 $[(F(1,48)=5.703, p=$ $.021), \eta^{2}=0.019$; see Figure 1]. However, evidence of an interaction of changes in the substance use involvement of social networks from $\mathrm{T} 1$ to $\mathrm{T} 3$ did not reach statistical significance $\left[(F(1,48)=3.159, p=.082), \eta^{2}=0.012\right]$.

\section{Figure 1}

Changes in network substance use involvement from T1 to T2 and T1 to T3 by relapse to alcohol and drugs or abstinence during the same time period

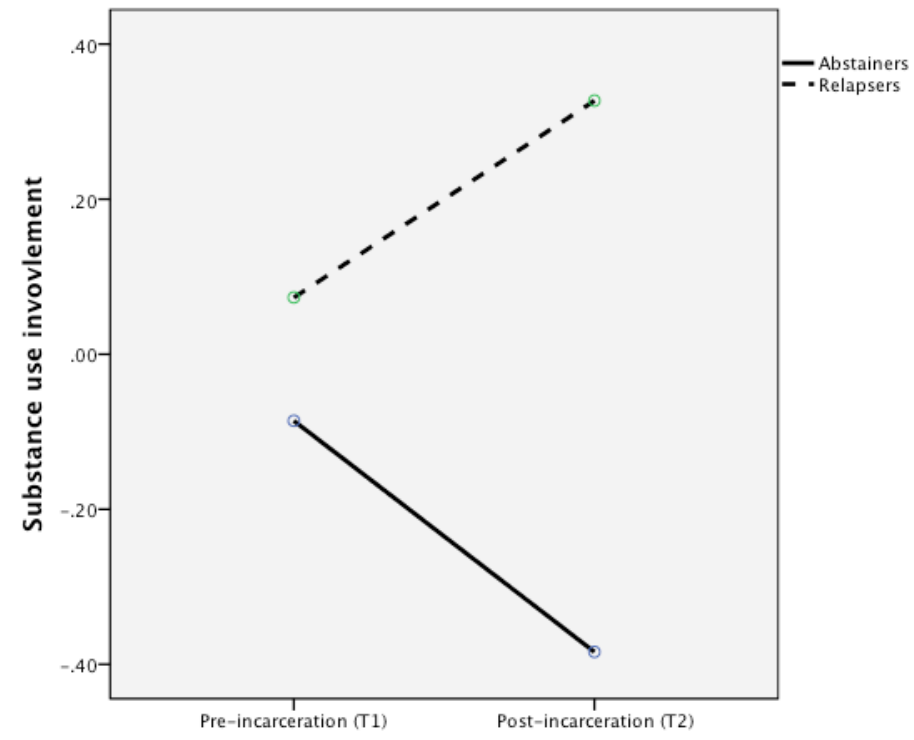

Discussion

The purpose of the current study was to examine how indices and component scores of the IPDA measure previously tested in a cocaine dependent, treatment-seeking sample replicated for recently incarcerated probationers with alcohol and drug use disorders. The similarity in the correlation matrices of the indices and the components is striking. For the concurrent and predictive validity analyses, the results from the present study actually present stronger evidence of the utility of the IPDA measure and the components derived by Zywiak et al. (2009). The findings from the original post hoc analyses and the parallel analyses conducted here also were very similar. As found by Zywiak et al. (2009), there was a significant interaction of relapse status and time predicting the substance use involvement index score. Results showed that many of the findings reported by Zywiak et al. (2009) replicated in the current sample, including the association between substance use involvement and drinking days, and general/treatment support and drug using days. One interesting divergence is that in the original study, the daily network size was inversely related to drug days ( $r=-.23)$, while in the present study the daily network size was directly related to drug days ( $r=.33$ ). In the current study, support for substance use was related to drug days; this was not found in the Zywiak et al. (2009) paper, but is

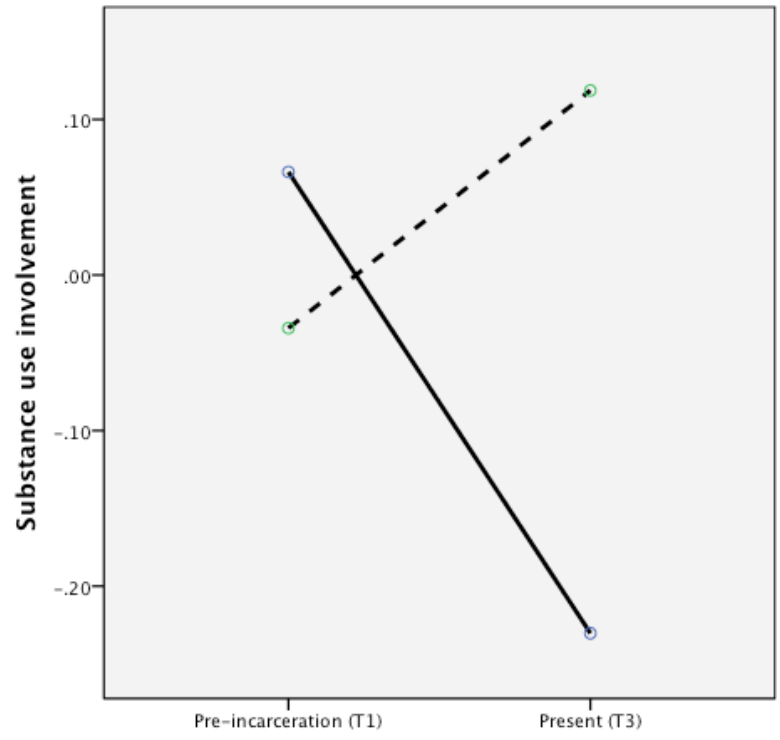

consistent with earlier findings reported by Beattie and Longabaugh (1999).

The findings from the current study provide encouraging evidence for the use of the IPDA measure to empirically examine the social networks of individuals with alcohol and drug use disorders. The association between social support/influence and substance use has been well documented (Longabaugh et al., 2010; Owens \& McCrady, 2014; Zywiak et al., 2002). The IPDA and its predecessors are the most commonly used measure of social support in substance use treatment research; however, the influence of social networks is complex and there is variability in how researchers use this measure to predict outcomes (e.g., Kelly, Stout, Magill, \& Tonigan, 2011; Owens \& McCrady, 2014; Project MATCH Research Group, 1997; Stout, Kelly, Magill, \& Pagano, 2012). This study showed that many of the indices and components used by Zywiak et al. (2009) have evidence of validity and provide valuable approaches to aggregating data from the IPDA to use in research and better understanding the role of social networks in models of substance use.

Clinically, the replication of the post hoc results from Zywiak et al. (2009) support the recommendation that clients with substance use disorders replace substanceinvolved persons with non-substance involved persons. These social network changes are one way of achieving 
treatment gains that endure over time. Further, the interaction of relapse and time for the first 30 days after incarceration (T2), but not for after the first month (T3), provides additional evidence that the first 30 days after release from jail may be the most important in terms of social network influences on individuals' alcohol and drug use. There are a large number of individuals involved with the criminal justice system and many meet criteria for a substance use disorder; these statistics, coupled with the current findings, suggest that the time proximal to release from jail presents a unique opportunity with which to intervene on individuals' social networks and substance use. One way to help adult male probationers' may be through the use of a brief motivational intervention that targets alcohol and drug use and social environments. By decreasing probationers' substance use it also may help to lower recidivism rates and help address the issue of the large criminal justice population. Clinicians could administer the IPDA to probationers to identify those that would benefit most from changes in their social networks.

There are a number of limitations associated with the current study. First, the small sample size likely resulted in decreased power and recruitment for the study was on a volunteer basis. Secondly, this was a cross-sectional and retrospective study, rather than a longitudinal design. Additionally, it is unknown if the changes in social networks were due to changes in the behaviors of social network members, or due to particular members leaving the network and new members coming into the network (e.g., heavy drug users leaving the network and more abstainers entering the network). Also, since only probationers who came into the probation office were recruited, how social network characteristics predict forensic outcomes could not be examined. Finally, except for the urine analysis, all measures were based on self-report and many were completed retrospectively; these factors could bias the information that was reported by participants.

There are many strengths associated with this study. First, individuals with substance use disorders who also are legally involved are an underrepresented sample in research. Further, many of the analyses of this study replicated those done by Zywiak et al. (2009), despite the smaller sample size, which suggest that these results may be representative of substance users in general. This is interesting given the differences between the two samples. The sample in the Zywiak et al. (2009) study was a treatment sample of 141 participants, equally representing men and women, with all clients meeting criteria for current cocaine dependence, and with Hispanic persons underrepresented $(6 \%)$ relative to the sample in the current study (56\%). Further the present sample is a forensic sample rather than a clinical sample, and drugs of choice were diverse with alcohol, marijuana, and opiates; all of which are more prevalent than cocaine. Finally, the replication of the interaction effects is particularly noteworthy given the difficulties sometimes encountered when the replication of matching effects is attempted (Project MATCH Research Group, 1999).
The current study showed support for indices and component scores to aggregate social network data from the IPDA. Research should continue to find ways to use this measure to operationalize and understand social support and networks. Given the utility of these scores, real-time software to score the IPDA should be developed to make it easier to use this measure in future research and clinical settings. As the IPA measure was successfully adapted into the IPDA for use with individuals with drug dependence, future studies may help to identify additional ways to adapt to the IPDA for other populations, such as social networks of individuals within complex systems, like jails or prisons.

Many of the results from concurrent and predictive analyses using the six variables of social support computed by Zywiak and colleagues (2009) were replicated in the current sample. Specifically, general support/support for treatment was found to be inversely related to concurrent number of days of alcohol and drug use. Additionally, substance use involvement was related to alcohol and drug use before and after incarceration. Social support has been identified as being a predictor of alcohol and drug use, which necessitates a reliable and valid measure for this construct. The findings from the current study showed that the IPDA is a promising method for measuring social support and could be utilized in both research and clinical settings.

\section{References}

Anton, R. F., O’Malley, S. S., Ciraulo, D. A., Cisler, R. A., Couper, D., Donovan, D. M., . . . \& Zweben, A. (2006). Combined pharmacotherapies and behavioral interventions for alcohol dependence. The COMBINE Study: A randomized clinical trial. Journal of the American Medical Association, 295(17), 2003-2017.

Beattie, M. C., \& Longabaugh, R. (1999). General and alcohol-specific social support following treatment. Addictive Behaviors, 24(5), 593-606.

First, M. B., Spitzer, R. L., Gibbon, M., \& Williams, J. B. W. (2002). Structured clinical interview for DSM-IVTR Axis I disorders, research version, patient edition (SCID-I/P). New York, NY, United States: Biometrics Research, New York State Psychiatric Institute.

Hides, L., Cotton, S. M., Berger, G., Gleeson, J., O’Donnell, C., Proffitt, T., . . . \& Lubman, D. I. (2009). The reliability and validity of the Alcohol, Smoking and Substance Involvement Screening Test (ASSIST) in first-episode psychosis. Addictive Behaviors, 34(10), 821-825.

IBM Corporation (2011). IBM SPSS statistics for Windows (Version 20.0) [Computer software]. Armonk, NY, United States: IBM Corp.

Kelly, J. F., Stout, R. L., Magill, M., \& Tonigan, J. S. (2011). The role of Alcoholics Anonymous in mobilizing adaptive social network changes: A prospective lagged mediational analysis. Drug and Alcohol Dependence, 114(2), 119-126. doi:10.1016/j.drugalcdep.2010.09.009 
Longabaugh, R., Wirtz, P. W., Zywiak, W. H., \& O’Malley, S. S. (2010). Network support as a prognostic indicator of drinking outcomes: The COMBINE Study. Journal of Studies on Alcohol and Drugs, 71(6), 837-846.

Miller, W. R. (Ed.). (2004). COMBINE monograph series, Volume 1. Combined behavioral intervention manual: A clinical research guide for therapists treating people with alcohol abuse and dependence. DHHS Publication No. (NIH) 04-5288. Bethesda, MD, United States: NIAAA.

Napper, L. E., Fisher, D. G., Johnson, M. E., \& Wood, M. M. (2010). The reliability and validity of drug users' self reports of amphetamine use among primarily heroin and cocaine users. Addictive Behaviors, 35(4), 350-354.

Owens, M. D., \& McCrady, B. S. (2014). The role of the social environment in alcohol or drug relapse of probationers recently released from jail. Addictive Disorders and Their Treatment, 13(4), 179-189. doi:10.1097/ADT.0000000000000039

Project MATCH Research Group. (1997). Matching alcoholism treatments to client heterogeneity: Project MATCH posttreatment drinking outcomes. Journal of Studies on Alcohol, 58(1), 7-29.

Project MATCH Research Group. (1998a). Matching alcoholism treatment to client heterogeneity: Project MATCH three-year drinking outcomes. Alcoholism: Clinical and Experimental Research, 22(6), 13001311.

Project MATCH Research Group. (1998b). Matching alcoholism treatments to client heterogeneity: Treatment main effects and matching effects on drinking during treatment. Journal of Studies on Alcohol, 59(6), 631-639.

Project MATCH Research Group. (1999). A study to remember: Response of the project MATCH research group. Addiction, 94(1), 66-69.

Stout, R. L., Kelly, J. F., Magill, M., \& Pagano, M. E. (2012). Association between social influences and drinking outcomes across three years. Journal of Studies on Alcohol and Drugs, 73(3), 489-497.

Tonigan, J. S., Miller, W. R., \& Brown, J. M. (1997). The reliability of Form-90: An instrument for assessing alcohol treatment outcome. Journal of Studies on Alcohol, 58(4), 358-364.

Weiss, R. D., Najavits, L. M., Greenfield, S. F., Soto, J. A., Shaw, S. R., \& Wyner, D. (1998). Validity of substance use self-reports in dually diagnosed outpatients. American Journal of Psychiatry, 155(1), 127-128.

Zywiak, W. H., Longabaugh, R., \& Wirtz, P. W. (2002). Decomposing the relationships between pretreatment social network characteristics and alcohol treatment outcome. Journal on Studies of Alcohol, 63(1), 114 121.

Zywiak, W. H., Neighbors, C. J., Martin, R. A., Johnson, J. E., Eaton, C. A., \& Rohsenow, D. J. (2009). The Important People Drug and Alcohol Interview: Psychometric properties, predictive validity, and implications for treatment. Journal of Substance Abuse Treatment, 36(3), 321-330. 